\title{
A SURFACE-MICROMACHINED SHEAR STRESS IMAGER
}

\author{
Fukang Jiang, Yu-Chong Tai, Bhusan Gupta, Rodney Goodman \\ Electrical Engineering 116-81, Caltech, Pasadena, CA 91125, USA \\ Steve Tung, Jin-Biao Huang, Chih-Ming Ho \\ MANE, UCLA, Los Angeles, CA 90024, USA
}

\begin{abstract}
A new MEMS shear stress sensor imager has been developed and its capability of imaging surface shear stress distribution has been demonstrated. The imager consists of multi-rows of vacuum-insulated shear stress sensors with a $300 \mu \mathrm{m}$ pitch. This small spacing allows it to detect surface flow patterns that could not be directly measured before. The high frequency response $(30 \mathrm{kHz})$ of the sensor under constant temperature bias mode also allows it to be used in high Reynolds number turbulent flow studies. The measurement results in a fully developed turbulent flow agree well with the numerical and experimental results previously published.
\end{abstract}

\section{INTRODUCTION}

The detection and control of turbulent flows have long been a dream of fluid mechanists because of its potential impact on aerospace industry [1-3]. Up to now, however, little has been done mainly because the unavailability of miniature devices whose sizes are comparable to the feature sizes in high-Reynolds-number turbulent flows. It is our goal to first demonstrate that distributed MEMS sensors and actuators can accomplish active drag reduction. To do this, the first task is to be able to collect, in real time, the information of distributed surface shear stress. This information will then be processed and passed to MEMS actuators to control and reduce the drag. Therefore, the first challenge to achieve the real-time detection of surface shear stress is to build a high-resolution MEMS shear stress imager. In this paper, we report the development of such an imager and the results of its field tests in turbulent flows.

\section{SKIN FRICTIONS AND VORTICES}

The basic understanding of turbulent flow is important for the proper design of the shear stress imager. When a flat plate is placed in a moving flow, it is subject to a skin friction drag. The drag is the integration of surface shear stress $\tau_{w}$, which is proportional to the flow velocity gradient near the surface, i.e.,

$$
\tau_{w}=\mu \frac{\partial u}{\partial y}
$$

where $u$ is the streamwise fluid velocity, $y$ is the axis normal to the surface, $\mu$ is the fluid viscosity. High skin friction drag has recently been linked to organized structures in turbulent flows. The high drag region is commonly observed near streamwise counter-rotating vortex pairs (Fig. 1). These vortices, which

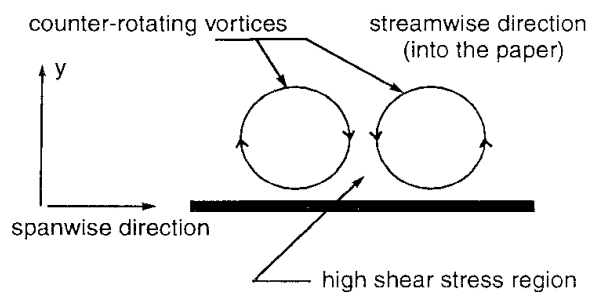

Fig. 1 Counter-rotating vortex pair. appear randomly in both space and time, bring high velocity fluids down to the walls and create local regions of high shear stress which significantly contributes to the total drag. Therefore, attempts to reduce drag have been focused on methods of either preventing the formation or mitigating the strength of these vortices.

The statistical size of the drag-inducing vortex pair streak decreases as the Reynolds number of the flow increases. For a typical airflow of $15 \mathrm{~m} / \mathrm{s}$ in wind tunnel, the Reynolds number is about $10^{4}$ and the vortex streaks have a mean width of about $1 \mathrm{~mm}$. The length of a typical vortex streak can be about $2 \mathrm{~cm}$, giving the streaks a 20:1 aspect ratio. The frequency of appearance of the streaks is approximately $100 \mathrm{~Hz}$. The life-time is around $1 \mathrm{~ms}$ [4].

\section{SHEAR STRESS SENSORS}

Many ways exist to do wall shear stress measurement [5]. Among them, the thermal method, which uses hot film sensors to determine shear stress indirectly, has many advantages over other techniques for real time flow measurement and control. For example, it can achieve high sensitivity while keeping the sensor size small. The traditional hot film sensors are thin metal film resistors on substrates, which is electrically heated in operation. Since only the heat convection responds to the shear stress change, it is desirable to thermally isolate the thin film resistor from the substrate to minimize the conductive heat loss, thus increasing the sensitivity. In the past, the only way to partially solve the problem was to use low thermal conductivity materials such as quartz for the substrate. Reasonable good sensitivity could be obtained only when such sensors are used to measure high thermal conductivity fluid such as water. However, they are not sensitive enough for the measurement in low thermal conductivity fluids such as air. Moreover, the size of traditional sensors is typically in the $\mathrm{mm}$ range [5]. This may be tolerable in measuring the mean value of shear stress, but is definitely not acceptable in shear stress imaging with reasonable spatial resolution.

Thanks to the development of surface micromachining technology, we can optimize both the materials and the structure of the sensors. Fig. 2 shows the cross-sectional structures of a few types of the micromachined shear stress sensors. Type I features a $2 \mu \mathrm{m}$ deep vacuum cavity with a $0.25 \mu \mathrm{m}$ thick polysilicon wire embedded in the nitride diaphragm. Here the vacuum cavity is designed for good thermal isolation of the diaphragm from the substrate [6]. Type II has a similar structure to type I except that the polysilicon wire is lifted $4 \mu \mathrm{m}$ above the diaphragm, thus achieving better thermal isolation. Type III is a conventional polysilicon bridge sitting on the solid substrate [7]. Type IV is basically a micromachined hot wire close to a wall as has been previously reported. The wire is a few microns above the substrate surface and is in the linear velocity distribution region so that it measures the wall shear stress instead of velocity [8]. All four types were fabricated on a single chip to ensure identical thermal and electrical properties of the sensor materials. Fig. 3 shows their calibration results in wind-tunnel. The output changes are proportional to the one third power of shear stress, which agrees with the heat transfer theory [5]. It is obvious that types I and II are the most sensitive ones. Moreover, type I has much simpler fabrication 


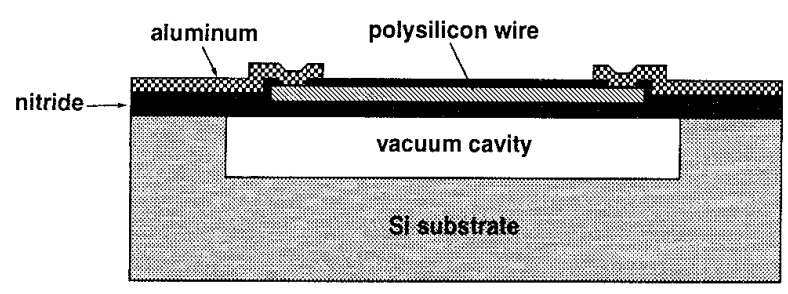

(a) Type I: Hot film.

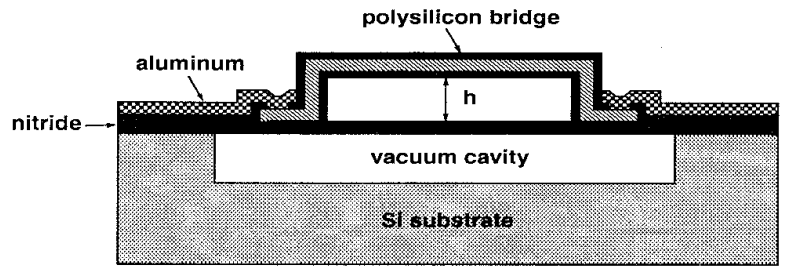

(b) Type II: Bridge over vacuum cavity.

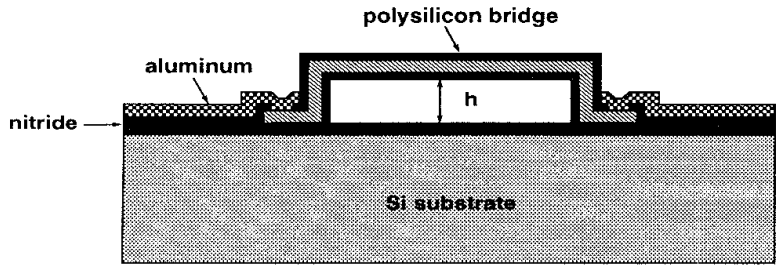

(c) Type III: Bridge over substrate.

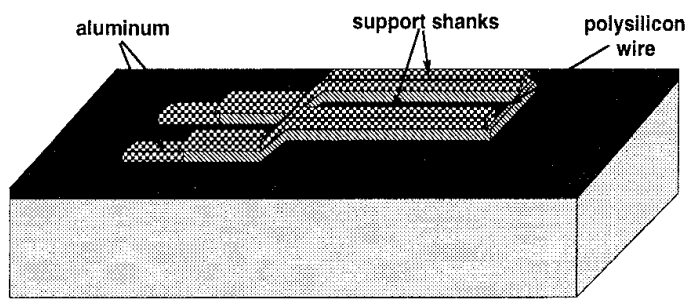

(d) Type IV: Hot wire.

Fig. 2 Structures of the sensors

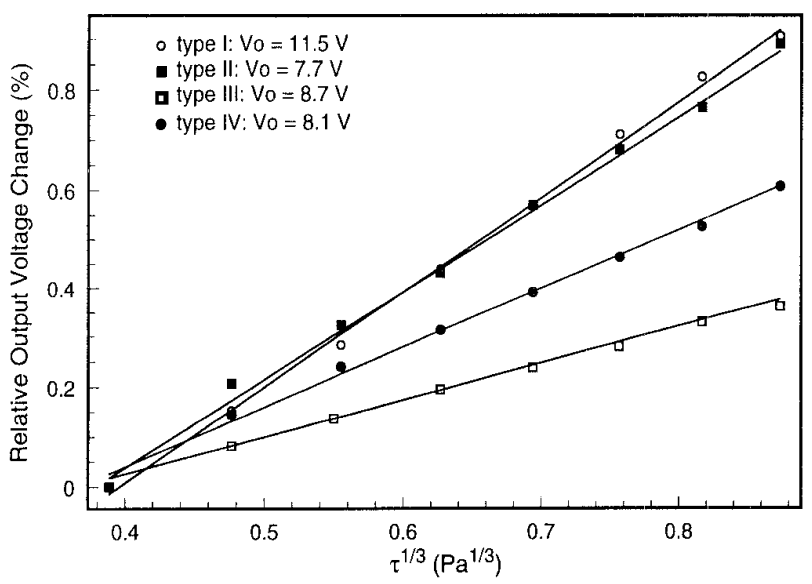

Fig. 3 Wind-tunnel calibration curves of sensors.

process than type II, it is therefore chosen as the building block of this generation of shear stress imaging chip.

After the structure is decided, the geometry of each layer is optimized to give maximum sensitivity. It is found that the sensitivity is higher for thinner diaphragm and larger aspect ratio polysilicon resistor (i. e. larger $l / w$, where $l$ is the length and $w$ the width). But the diaphragm can not be too thin as it would otherwise break during fabrication or operation. $l$ is limited by the size of the whole device, which is at most $300 \mu \mathrm{m}$ for the application in shear stress imaging. Therefore, $l$ is chosen to be $150 \mu \mathrm{m}$. Also $w$ is limited by the photolithography and etching technology. In this case, it is designed to be $3 \mu \mathrm{m}$ to ensure good uniformity.

\section{SHEAR STRESS IMAGER}

Fig. 4 shows the photomicrograph of the $2.85 \mathrm{~cm} \mathrm{x} 1.0 \mathrm{~cm}$ imaging chip using type I shear stress sensor. It is specifically designed for the study in the turbulent flow with Reynolds number near $10^{4}$.There are two identical sensor rows $5 \mathrm{~mm}$ apart, parallel to the broad side of the chip. This vertical pitch

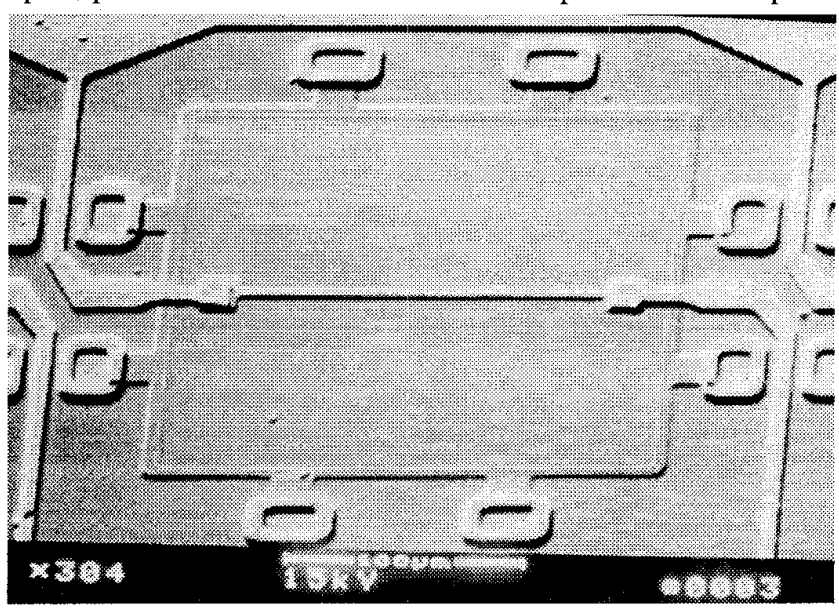

(a) SEM of a single sensor.

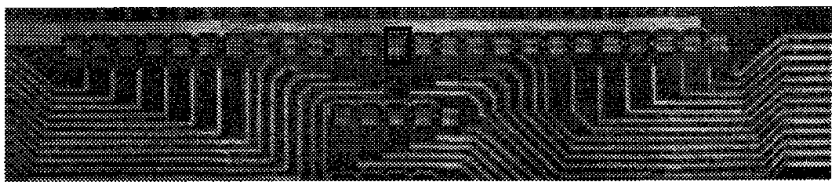

(b) One row of 25 sensors

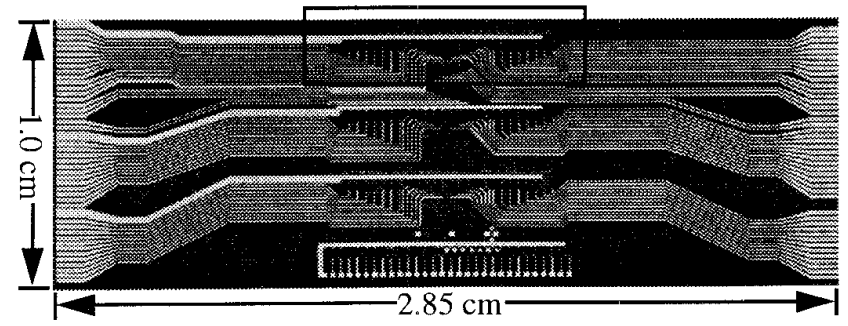

(c) The shear stress imaging chip.

Fig. 4 Pictures.

is chosen such that at least four data points can be taken from a streak in the streamwise direction. Each row has 25 sensors with $300 \mu \mathrm{m}$ pitch, which is already the minimum for this type of sensor. It should give at least three data points from a streak in the spanwise direction and be able to catch more than one streaks. The $1 \mathrm{~cm}$ spacing between the sensors and the left and right edges of the chip are necessary to avoid the upstream bonding wires from interfering with the downstream sensors.

The fabrication process of the shear stress imager starts with the deposition of $4000 \mathrm{~A}$ of LPCVD low stress silicon nitride. In some of the area, the nitride is removed by plasma 


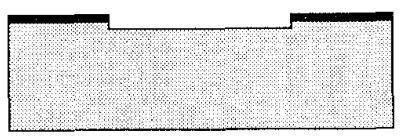

1. Deposit and pattern LPCVD nitride.

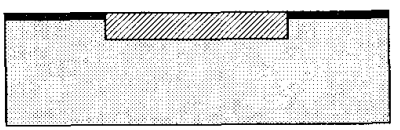

2. Local oxidation (LOCOS).

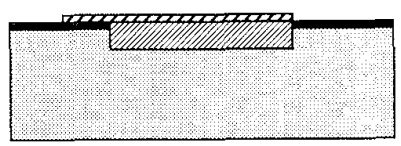

3. Deposit and pattern PSG.

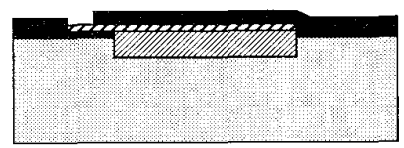

4. Deposit thick nitride and open etch holes.

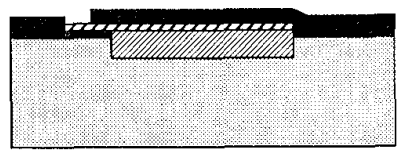

5. $49 \% \mathrm{HF}$ etches oxide and PSG.

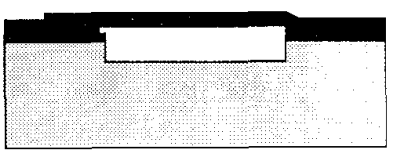

6. Seal the cavity.

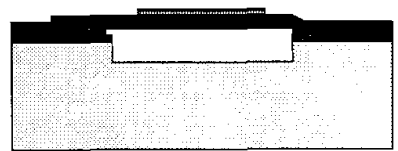

7. Deposit, dope and pattern polysilicon.

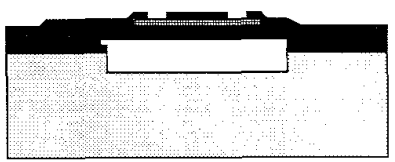

8. Deposit thin nitride and open contact holes.

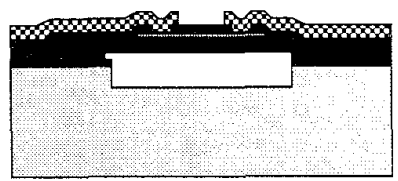

9. Metallization

Fig. 5 Fabrication process of the shear stress imager

with a little over-etch to give a 7000-8000A cavity. Then the wafer is put in oxidation furnace to grow $1.7 \mu \mathrm{m}$ thick oxide in the cavity. After short time etch in BHF to remove the oxidized nitride, 4000A PSG is deposited, patterned and annealed to form the sacrificial layer etching channel. Next, $1.2 \mu \mathrm{m}$ of LPCVD low stress nitride is deposited as the diaphragm material. Etching holes are opened to expose the end of the PSG etching channel, followed by a $49 \% \mathrm{HF}$ etching to completely remove the PSG and thermal oxide underneath the diaphragm. The cavity is then sealed by LPCVD LTO and nitride deposition at a vacuum of 200 mTorr. The sealing materials on the diaphragm are removed by plasma and BHF etching to minimize the diaphragm thickness. $4000 \mathrm{~A}$ polysilicon layer is deposited, doped, annealed and patterned to form the resistor on diaphragm. Another $2000 \mathrm{~A}$ of low stress nitride is deposited to passivate the resistor. After the contact hole opening, metallization is done and the wafer is diced to $2.85 \mathrm{~cm} \times 1 \mathrm{~cm}$ chips. The fabrication process flow is shown in Fig. 5.

\section{PACKAGING, BIASING AND CALIBRATION}

The package for the imaging chip is a fine-line $\mathrm{PC}$ board with a recess in the center so that the imaging chip can be flush-mounted. The chip and the PC board are electrically connected by wire bonding. The electrical leads are soldered on the backside of the PC board. The PC board is then flushmounted on a specially-made plug which fits into the wall of the wind-tunnel (Fig. 6), with the sensor row perpendicular to the flow direction.

The wind tunnel supplies a two-dimensional channel flow. The channel is $4.87 \mathrm{~m}$ long with an cross-sectional area of $60 \mathrm{~cm} \times 2.5 \mathrm{~cm}$. The walls of the channel are constructed of $2.5 \mathrm{~cm}$ thick Plexiglas and supported by a steel frame. An axial blower powered by a DC source supplies the air flow in the channel. At the highest blower speed, the centerline velocity in the channel is about $25 \mathrm{~m} / \mathrm{s}$. Hot-wire velocity measurements at $10 \mathrm{~m} / \mathrm{s}$ indicate that the channel consists of a laminar

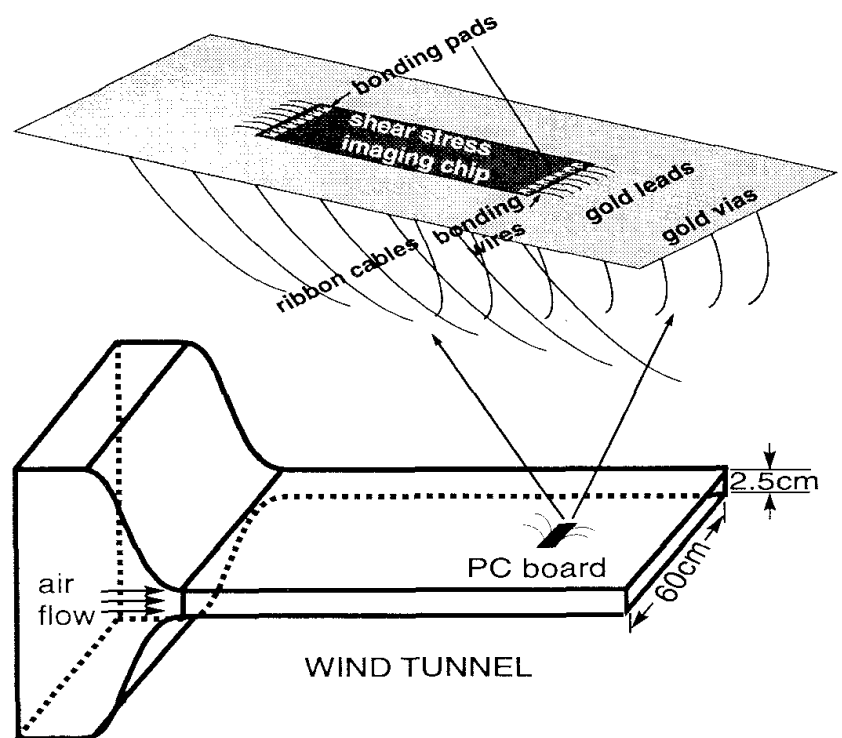

Fig. 6 Imaging chip package and wind tunnel setup.

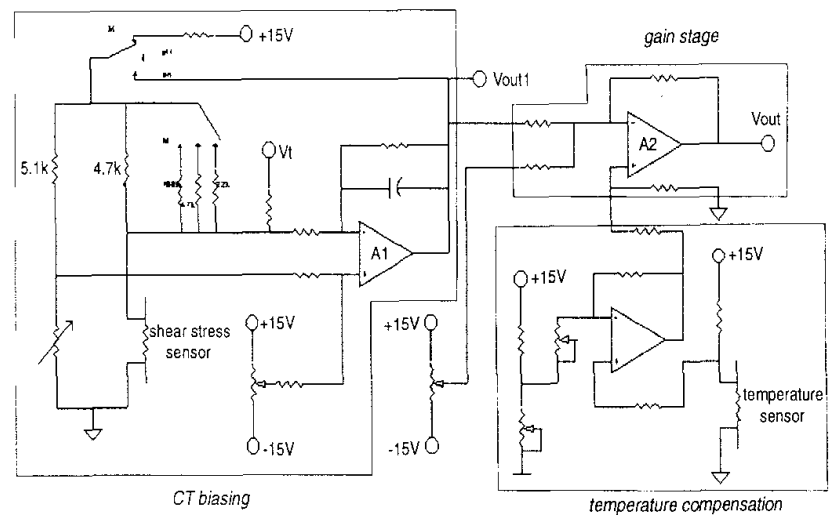

Fig. 7 Constant temperature biasing circuit,

gain stage and temperature compensation stage.

entrance flow region which gradually transforms into a fullydeveloped turbulent flow in the downstream $2 / 3$ portion of the channel. All calibration and testing of the imaging chip is carried out in this region of the channel.

In our experiments, the sensors are biased in constant temperature (CT) mode. Although it is more complicated than the constant current mode, it can achieve much higher frequency bandwidth which is crucial in turbulence measurement. Therefore, arrays of CT circuits and gain stages have been made on PC boards using op-amps and discrete components (Fig. 7). The dc offset of the outputs can be adjusted individually, but the gain is fixed to be 10 . A computer-controlled data acquisition system is used to measure all the outputs simultaneously.

Before the sensors are used to measure the shear stress distribution, their de outputs are calibrated against known wall shear stress levels which are calculated from the centerline velocity by using the following relationship [9],

$$
\begin{gathered}
\frac{U_{t}}{U_{c}}=0.1079(\operatorname{Re})^{-0.089} \\
\operatorname{Re}=\frac{U_{c} d}{V} \\
U_{t}=\left(\frac{\tau_{w}}{\rho}\right)^{0.5}
\end{gathered}
$$


where $\boldsymbol{R} \boldsymbol{e}$ is the Reynolds number, $\boldsymbol{U}_{\boldsymbol{c}}$ is the centerline velocity of the channel, $U_{t}$ is the friction velocity, $d$ is the half width of the channel $(=1.27 \mathrm{~cm}), v$ is the kinematic viscosity of air, $\tau_{\mathrm{w}}$ is the wall shear stress, and $\rho$ is the density of air.

Fig. 8 shows the calibration results for 10 sensors in a row. Although each sensor has different offset, the trend of all curves are almost the same. Polynomial fitting is performed on each curve to extract the fitting parameters for later use in real data processing.

It is important to note that the dc outputs of the sensors are sensitive to the fluid temperature because the shear stress sensor is essentially a thermal sensor with temperature coefficient of resistance around $0.1 \% /{ }^{\circ} \mathrm{C}$. This dc drift can be compensated by measuring the sensor temperature sensitivity and monitoring the fluid temperature change using a temperature sensor. Fig. 9 shows that an order of magnitude of improvement on the thermal stability has been achieved by the compensation circuit given in Fig. 7. Here the temperature sensor is just another shear stress sensor operated at very low power such that the self-heating is negligible.

To confirm that the sensors have enough bandwidth to pick up all the information in turbulent flow under investigation, the frequency response of a sensor in CT mode is measured using a sinusoidal electrical testing signal $v_{t}$. It has been shown that, if the sensor has a single time constant, the output of the CT circuit in Fig. 6 can be expressed as follows [10],

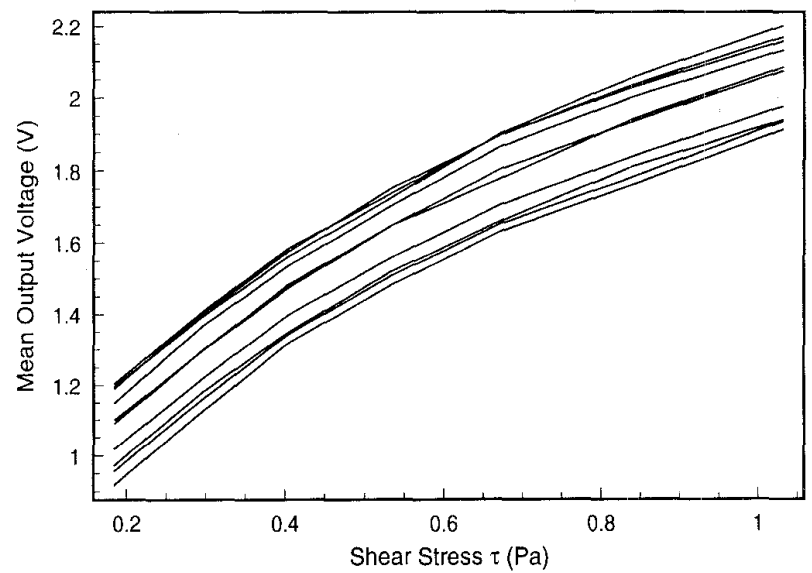

Fig. 8 Calibration curves of 10 sensors in a row.

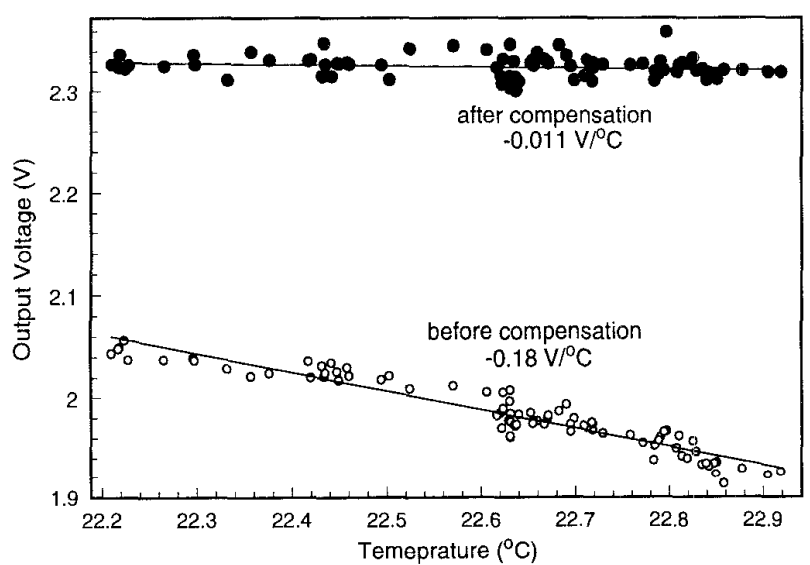

Fig. 9 Typical temperature sensitivities before and after temperature compensation of a shear stress sensor.

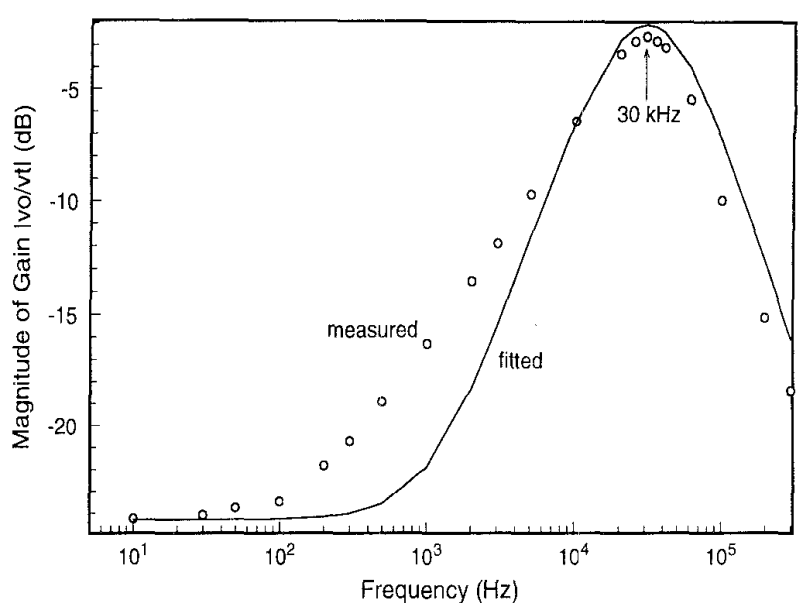

Fig. 10 Frequency response of a CT sensor.

$$
\frac{G_{v}\left(1+\frac{s}{\omega_{1}}\right) v_{t}+S_{\tau} \tau_{w}}{1+\frac{s}{\xi \omega_{2}}+\left(\frac{s}{\omega}\right)^{2}}
$$

where $\boldsymbol{G}_{\boldsymbol{v}}$ and $\boldsymbol{S}_{t}$ are the low frequency voltage gain and shear stress sensitivity, $\omega_{1}$ is approximately the cut-off frequency of the sensor in CC mode, $\xi$ is the damping factor and $\omega_{2}$ is the bandwidth of the sensor in CT mode if the circuit is in critical damping. Fig. 10 shows that this bandwidth reaches $30 \mathrm{kHz}$, which is sufficient for the turbulent flow under study. The deviation between the experimental data and the fitted curve is due to the fact that a real sensor has multiple thermal time constants.

\section{SHEAR STRESS IMAGING}

For real-time shear stress imaging, the output voltage is sampled at $10 \mathrm{kHz}$ and converted to shear stress signal based on the calibration performed previously. In order to establish the credibility of the imaging chip, the turbulence statistics calculated from the shear stress fluctuations recorded by a single shear stress sensor are compared to previously established results. Fig. 11 shows the comparison in terms of the normalized RMS level, the skewness factor, and the flatness factor. It is obvious that the present results agree very well with previous studies in all three areas [11-13]. In addition, the present statis-

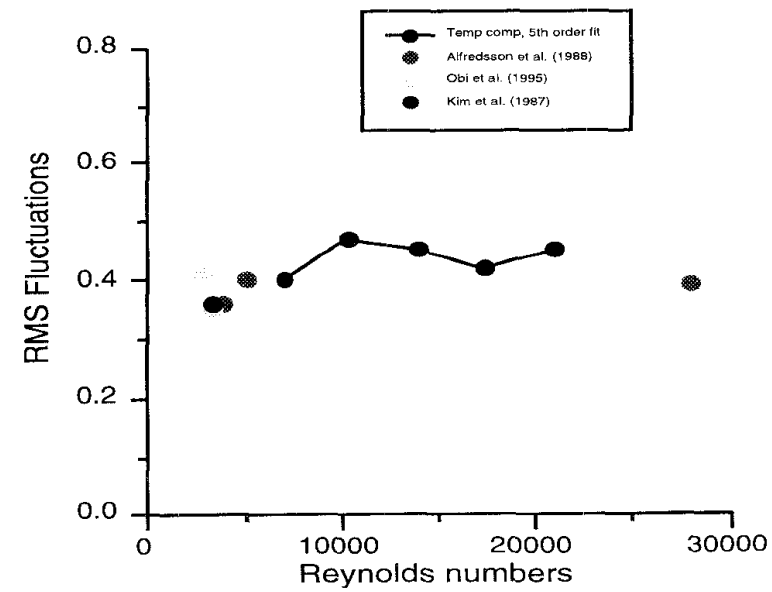

(a) RMS fluctuation 


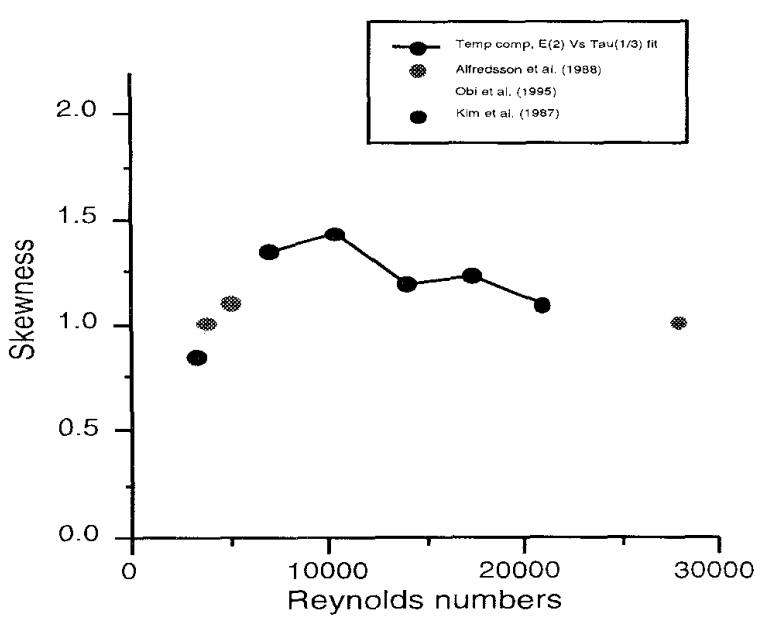

(b) Skewness factor.

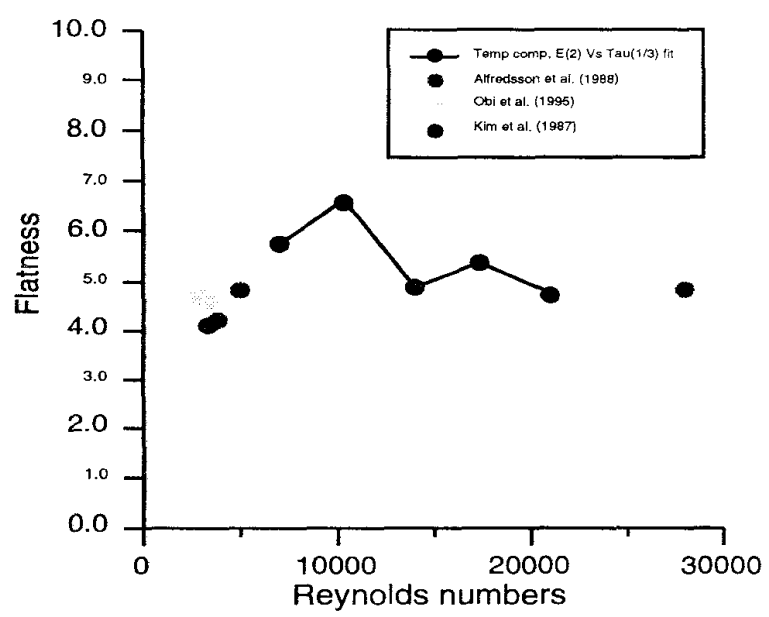

(c) Flatness factor.

Fig. 11 Turbulence statistics based on the shear stress fluctuations recorded by the micro shear stress sensors on the imaging chip.

tics appear to be independent of the Reynolds number which is predicted by turbulence theory.

Next, the instantaneous turbulent shear stress distributions of the channel were recorded by using one of the sensor rows on the imaging chip. Fig. 12 shows the contour plots of the instantaneous shear stress distributions at two different centerline velocities. The white streaky structures in the plots represent regions of high shear stress on the wall of the channel where the imaging chip is located. They are caused by the presence of near-wall streamwise vortices which bring high momentum fluid from the freestream to the wall. Due to the small-scale nature of these structures, previously experiments in turbulent boundary layers have only succeeded in qualitatively demonstrating their existence without obtaining any quantitative information. This is the first time that the instantaneous shear stress levels associated with the near-wall structures are recorded.

The contour plots in Fig. 12 indicate that the scales of the streaks are different at different centerline velocities. The streaks in the high-speed $(20 \mathrm{~m} / \mathrm{s})$ case appear to be thinner and more densely packed than those in the low-speed $(8 \mathrm{~m} / \mathrm{s})$ case. Similar phenomena have also been observed in previous experiments. Based on real-time movies generated from the contour

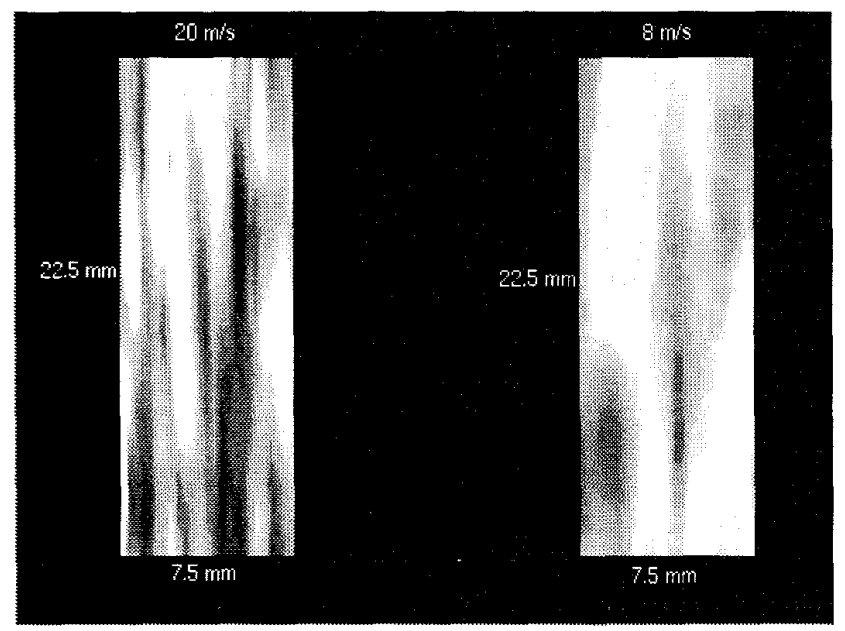

Fig. 12 Contour plot of shear stress distribution.

plots similar to the ones in Fig. 12, the average streamwise length, the average spanwise scale, and the average spanwise spacing of the streaks at three different centerline velocities are estimated and shown in Fig. 13. Once again, the present results

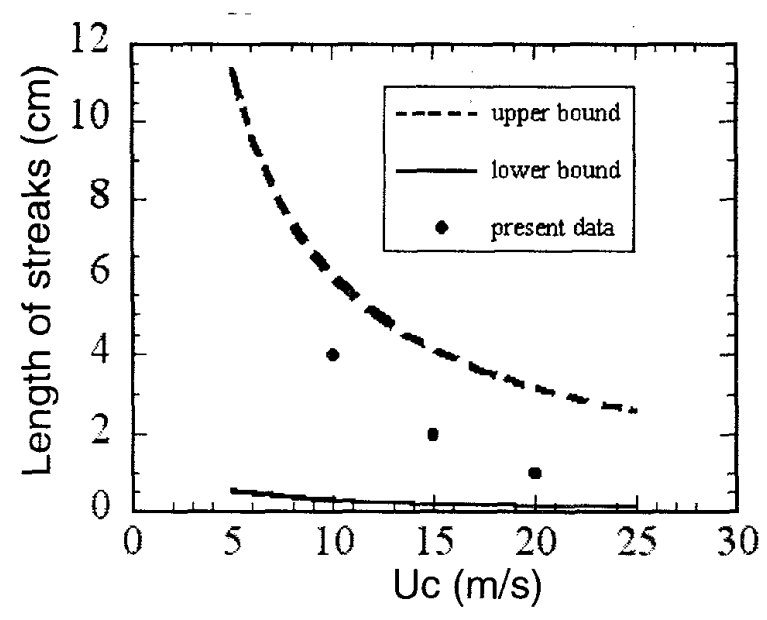

(a) The length of streaks.

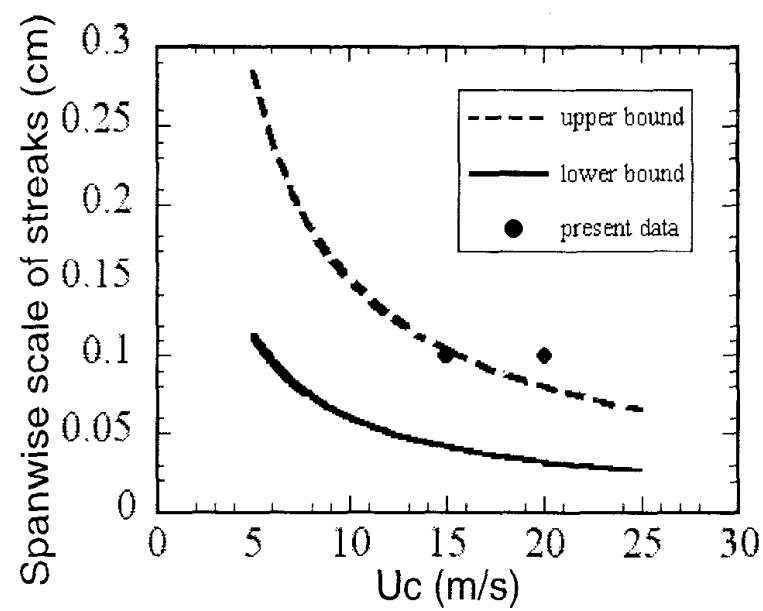

(b) The spanwise scale of streaks. 


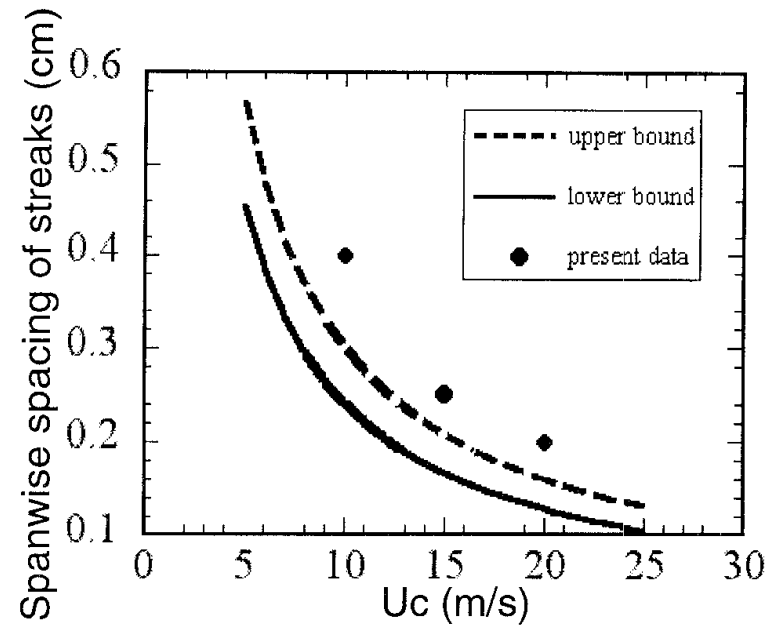

(c) The spanwise spacing of streaks.

Fig. 13 Scales of the near-wall streaky structures at different Reynolds numbers.

agree well with previous studies. The average length of the present streaks falls within the upper and lower bounds of the established results. The average spanwise scale and spacing of the present streaks are either on or slightly higher than the upper bound.

\section{CONCLUSION}

A new MEMS shear stress imager has been developed and its capability of imaging shear stress distribution has been demonstrated. It will allow new studies of micro fluid mechanics in turbulent flows. Our future research will then include using the device to study the interaction between turbulent flows and micro actuators [14] for the goal of turbulent drag reduction.

\section{ACKNOWLEDGMENTS}

This work is jointly supported by AFOSR and ARPA. The authors would like thank Mr. Trevor Roper for the help in processing and Dr. Chang Liu for the help in imager design.

\section{REFERENCES}

[1] P. R. Bandyopadhyay, "Development of a Microfabricated Surface for turbulence Diagnostics and Control", ASME Application of Microfabrication to Fluid Mechanics, Chicago (1994), pp. 67-74.

[2] D. M. Bushnell, "Turbulent Drag Reduction for External flows", AIAA Papaer No. 83-0227, 1983.

[3] P. Moin, J. Kim, H. Choi, "On the Active Control of WallBounded Turbulent Flows", AIAA Paper No. 89-0960, 1989.

[4] J. Cantwell, "Organized Motion in Turbulent Flow", Annual Review of Fluid Mechanics, vol. 13 (1981), pp. 475515 .

[5] R. J. Goodstein, Fluid Mechanics Measurements, Chapter 11, Hemisphere Publish Corp., 1983, pp. 559-615.

[6] C. Liu, Y. C. Tai, J. B. Huang, C. M. Ho, "Surface Micromachined Thermal Shear Stress Sensor", ASME Application of Microfabrication to Fluid Mechanics, Chicago (1994), pp. 9-15.

[7] Y. C. Tai, R. S. Muller, "Lightly-Doped Polysilicon Bridges as Flow Meter", Sensors and Actuators, Vol. 15(1), 1988, pp. 63-75.

[8] F. Jiang, Y. C. Tai, J. B. Huang, C. H. Ho, "Polysilicon Structures for Shear Stress Sensors", Digest IEEE TENCON'95, Hong Kong, Nov. 1995, pp.

[9] A. K. M. F. Hussain and W. C. Reynolds, "The mechanics of a perturbation wave in turbulent shear flow", $A F O S R$ Scientific Report 70-1655TR, 1970.

[10] F. Jiang, Y. C. Tai, C. H. Ho, R. Karan, M. Garstenauer, "Theoretical and Experimental studies of the Micromachined Hot-Wire Anemometers", Tech. Digest 1994 IEDM, San Francisco, 1994, pp. 139-142.

[11] P. H. Ferredoxin, A. V. Johansson, J. H. Haritonidis and H. Eckelman, "The fluctuating wall-shear stress and the velocity field in the viscous sublayer", Physics of Fluids, Vol. 31, 1988, pp.1026-1033.

[12] S. Obi, K. Inoue, T. Furukawa and S. Masuda, "Experimental study on the statistics of wall shear stress in turbulent channel flows", Tenth symposium on turbulent shear flows, The Pennsylvania State University, Vol. 1, 1995, pp. 5-19 5-24.

[13] J. Kim, P. Moin and R. Moser, "Turbulence statistics in fully developed channel flow at low Reynolds number", Journal of fluid Mechanics, Vol. 177, 1987, pp. 133-166.

[14] T. Tsao, C. Liu, Y. C. Tai, C. H. Ho, "Micromachined Magnetic Actuator for Active Fluid Control", ASME Application of Microfabrication to Fluid Mechanics, Chicago (1994), pp. 31-38. 